\title{
Die wichtigsten Änderungen der S3-Leitlinie der Deutschen Gesellschaft für Gastroenterologie, Verdauungs- und Stoffwechselkrankheiten (DGVS) zur Prophylaxe, Diagnostik und Therapie der Hepatitis-B-Virusinfektion im Überblick
}

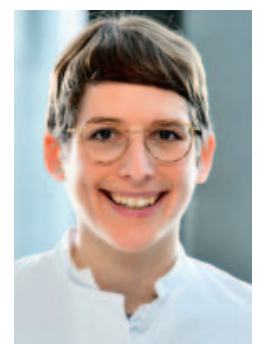

Lisa Sandmann

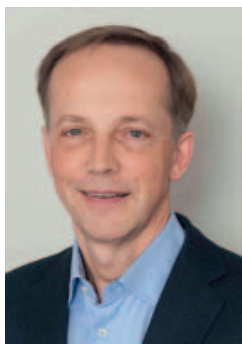

Jörg Petersen

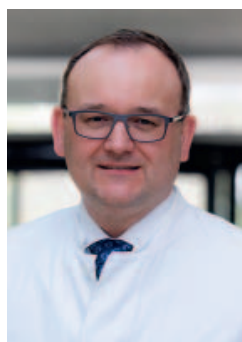

Markus Cornberg
Korrespondenzadresse

Prof. Dr. Markus Cornberg

Klinik für Gastroenterologie, Hepatologie und Endokrinologie

Medizinische Hochschule Hannover, Carl-Neuberg-Str. 1,

30625 Hannover, Deutschland

Tel.: +49/5 11/5326821

cornberg.markus@mh-hannover.de
Bibliografie

Z Gastroenterol 2021; 59: 641-643

DOI $10.1055 / a-1498-2680$

ISSN 0044-2771

(c) 2021. Thieme. All rights reserved.

Georg Thieme Verlag KG, Rüdigerstraße 14,

70469 Stuttgart, Germany
In den letzten Jahren wurden gut etablierte Leitlinien der Deutschen Gesellschaft für Verdauungs- und Stoffwechselkrankheiten (DGVS) zur Hepatitis-B-Virusinfektion (HBV) publiziert [1, 2]. Die letzte Veröffentlichung erfolgte 2011, sodass eine Überprüfung und Aktualisierung nun dringend erforderlich war. Es wurden 165 Empfehlungen aus 7 verschiedenen Bereichen erneut beurteilt, auf ihre weitere Gültigkeit überprüft und entsprechend der aktuellen Evidenzlage adaptiert oder neu erstellt (geprüft 64, modifiziert 57, neu 44). Zur Erstellung evidenzbasierter Empfehlungen wurden weitere seit 2011 veröffentlichte Leitlinien, Metaanalysen und randomisierte Studien berücksichtigt. Insbesondere wurden Leitlinienadaptationen der internationalen EASLund AASLD-Leitlinien zur Hepatitis B [3-5], der WHO-Leitlinie zur Prävention einer Mutter-Kind-Übertragung einer HBV-Infektion [6], der DGVS S3-Leitlinie zum hepatozellulären Karzinom (HCC) [7], der EASL-Leitlinie zur nichtinvasiven Fibrosebestimmung [8] sowie der DGVS S3-Leitlinie zur Hepatitis C $[9,10]$ vorgenommen. Nachfolgend sind die wichtigsten Neuerungen der aktuellen Leitlinie adressiert:

Diagnostik der Hepatitis-B-Virusinfektion: Im Bereich der Diagnostik ergeben sich einige wichtige neue Ergänzungen. In einem eigenen Abschnitt wird auf den Einsatz der nichtinvasiven Verfahren zur Beurteilung der Leberfibrose eingegangen. So kann für die Beurteilung der Leberfibrose zur Therapieindikationsstellung die transiente Elastografie eingesetzt werden, die sowohl für die fortgeschrittene Fibrose/Zirrhose als auch für fehlende Fibrose eine hohe Prädiktionsgenauigkeit besitzt. Die Leberbiopsie ist weiterhin hilfreich zur Einstufung der entzündlichen Aktivität und kann bei Patienten mit chronischer HBV-Infektion durchgeführt werden, wenn sich hieraus Konsequenzen für Diagnose, Verlaufsbeurteilung oder Therapie ableiten lassen. Unverändert ist bei allen Schwangeren ein HBsAg-Screening empfohlen. Die aktuelle Leitlinie empfiehlt jedoch ein möglichst frühes Screening, idealerweise zu Beginn der Schwangerschaft und nicht erst ab der 32. Schwangerschaftswoche, wie in den Mutterschaftsrichtlinien empfohlen. Auf diese Weise kann im Falle einer Therapieindikation ein ausreichend früher Therapiebeginn ermöglicht werden, sodass durch den Abfall der HBVDNA bis zur Geburt ( $<200000 \mathrm{IU} / \mathrm{ml}$ ) das Risiko einer vertikalen Transmission hinreichend reduziert werden kann.

Indikation zur Therapie: Die Nomenklatur der 4 Phasen der chronischen HBV-Infektion wurde modifiziert. Es wird zwischen HBeAg-positiver Infektion, HBeAg-positiver Hepatitis, HBeAgnegativer Infektion und $\mathrm{HBeAg-negativer} \mathrm{Hepatitis} \mathrm{unterschie-}$ den. Eine klare Therapieindikation besteht bei einer Hepatitis, insbesondere bei Leberfibrose oder Leberzirrhose. Neu ist die Empfehlung zur antiviralen Therapie bei Vorliegen eines HCC und nachweisbarer Viruslast.

Das kontroverse Thema der Behandlung von Patienten mit HBeAg-positiver Infektion (früher als immuntolerante Patienten bezeichnet) wird intensiv diskutiert. Bei Patienten mit HBeAgpositiver chronischer HBV-Infektion kann bei einem Alter > 30 Jahren und hochnormalen Transaminasen analog zu den Empfehlungen der EASL eine antivirale Therapie begonnen wer- 
den. Diese Patienten weisen vermehrt histologische Veränderungen auf und zeigen ein erhöhtes Risiko für die Entwicklung eines HCC.

Zudem hat die medikamentöse antivirale Therapie von Schwangeren mit hoher HBV-DNA > $200.000 \mathrm{IU} / \mathrm{ml}$ zur Verhinderung der vertikalen Transmission auf das Neugeborene einen höheren Stellenwert erhalten, da die Effektivität der Therapie mit hoher Evidenz gezeigt wurde. Dabei sollte auf einen ausreichend frühen Beginn (nach dem ersten Trimenon und vor der 28. Schwangerschaftswoche laut WHO-Leitlinie) geachtet werden, sodass eine ausreichende Behandlungszeit bis zur Entbindung zur Senkung der Viruslast erreicht werden kann. Hier besteht eine Diskrepanz zur aktuellen Mutterschaftsrichtlinie, die ein HBsAgScreening erst nach der 32. Schwangerschaftswoche empfiehlt. Hierdurch werden Mütter mit einer Indikation für eine antivirale Therapie zu spät diagnostiziert. Erfolgt die Bestimmung von HBsAg zu Beginn der Schwangerschaft, kann die infizierte Mutter rechtzeitig einem Experten zugewiesen, therapiert und eine chronische Infektion des Neugeborenen verhindert werden.

Unter immunsuppressiver Therapie (z. B. Chemotherapie, Rituximab, Anti-TNF) kann eine inaktive oder „ausgeheilte“ HBV-Infektion in lebensbedrohlicher Form reaktiviert werden. Die Prävention einer HBV-Reaktivierung ist daher ein zentrales Thema in neuen Leitlinien. Eine prophylaktische Therapie kann die Reaktivierung verhindern. Dezidierte Entscheidungshilfen sind in der Abbildung 3.13 dargestellt.

Therapie der chronischen Hepatitis-B-Virusinfektion: Standard sind direkt antiviral wirkende Nukleosid-/Nukleotidanaloga (NA) mit hoher Resistenzbarriere wie Entecavir (ETV) oder Tenofovir (TDF oder TAF). Eine Therapie mit Lamivudin kann bei optimalem Therapieansprechen fortgeführt, aber nicht neu begonnen werden. Adefovir sollte nicht mehr eingesetzt werden. Generell ist bei der Wahl des NA auf Komorbiditäten, das Stadium der Lebererkrankung, Begleitumstände wie bspw. Schwangerschaft, Kinderwunsch oder Transplantation zu achten. Eine Therapie mit TDF sollte bei einem Abfall der glomerulären Filtrationsrate (GFR), beim Auftreten einer Tubulopathie, bei einer nüchtern gemessenen Hypophosphatämie $<1 \mathrm{mg} / \mathrm{dl}$ und bei Frakturrisiko auf ein anderes NA (TAF oder ETV) umgesetzt werden. Die Therapie mit pegyliertem Interferon alfa wird unverändert in der Leitlinie beschrieben und sollte bei allen Patienten als mögliche Therapieoption geprüft werden, da es sich um eine zeitlich begrenzte antivirale Therapie handelt und bei einigen Patienten der Verlust von HBsAg (funktionelle Heilung) einen realistischen klinischen Endpunkt darstellt. Eine Kombination beider Therapiekonzepte wird hingegen ausdrücklich nicht empfohlen.

Im Gegensatz zur Interferon-Behandlung ist eine Therapie mit NA meist eine Dauertherapie und bei HBeAg-negativen Patienten sollte die Therapie erst nach Erreichen eines HBsAg-Verlusts abgesetzt werden. Allerdings kann in Einzelfällen und unter gesicherter Nachsorge die NA-Therapie früher beendet werden (siehe Abschnitt 3.8.4 der Leitlinie). Geeignete Kandidaten sind Patienten ohne fortgeschrittene Fibrose mit einer langjährigen HBVDNA-Suppression (>3 Jahre). Ein engmaschiges Kontrollintervall von 4 Wochen im ersten Jahr nach Absetzen der Therapie ist Voraussetzung. Insgesamt ist eine funktionelle Heilung mit den aktuellen Therapien selten. Neue Substanzen werden zurzeit in zahlreichen Phase-I- bis -II-Studien untersucht und es zeigen sich vielversprechende Ergebnisse.

Organtransplantation: Zur Vorbeugung einer Reinfektion des Spenderorgans nach Lebertransplantation soll eine duale Prophylaxe mit Hepatitis-B-Immunglobulin (HBIG) und einem hochpotenten NA erfolgen. Dies war in dieser Form bereits in der vorherigen Leitlinie aufgeführt. Neu ist, dass diese Kombinationsprophylaxe nicht mehr zwingend unbegrenzt empfohlen wird. Anhand prospektiver Studiendaten konnte belegt werden, dass nach initial effektiver dualer Prophylaxe (HBsAg negativ, Anti-HBs positiv, HBV-DNA negativ) die Prophylaxe mit einer alleinigen oralen Therapie mit einem potenten NA ebenso effektiv wie die bisher etablierte lebenslange Kombinationsprophylaxe ist. Als Risikofaktoren für eine Reinfektion unter NA-Monoprophylaxe wurde neben einer hohen HBV-DNA zum Transplantationszeitpunkt auch das Vorliegen einer HBV/HDV- oder HBV/HIV-Koinfektion oder eines HCC vor Transplantation identifiziert (Tabelle 4.3). In diesen Fällen sollte die Kombinationsprophylaxe fortgeführt werden. Neben der Lebertransplantation werden in der aktuellen Leitlinie Empfehlungen zur Kontrolle und Prophylaxe vor und nach Transplantation anderer Organe inkl. Stammzelltransplantation ausführlich erläutert. Die aktuellen Empfehlungen sind in Tabelle 4.10 zusammengefasst.

Immunprophylaxe: Wichtige neue Punkte im Bereich der Prophylaxe beziehen sich neben der bereits mehrfach erwähnten Reduktion einer Mutter-zu-Kind-Transmission auch auf die Indikation bzw. das Vorgehen bei chronischer HBV-Infektion und Tätigkeit im Gesundheitswesen. Bei einer Viruslast von $<200 \mathrm{IU} / \mathrm{ml}$ ist in der Regel von keinem erhöhten Übertragungsrisiko auszugehen, Kontrollen sollten jedoch alle 3 Monate erfolgen. Eine Viruslast von > $20000 \mathrm{IU} / \mathrm{ml}$ ist nicht mit einer übertragungsträchtigen Tätigkeit zu vereinbaren, sodass eine Therapie oder Änderung der Tätigkeit besprochen werden muss. Im Zwischenbereich (200$20000 \mathrm{IU} / \mathrm{ml}$ ) soll eine individuelle Bewertung des Übertragungsrisikos erfolgen.

Relevante Koinfektionen: Bei der Hepatitis-D-Virusinfektion bezieht sich die Leitlinie aktuell weiterhin auf die antivirale Therapie mit pegyliertem Interferon alfa. Ein zeitnahes Addendum zur aktualisierten Therapieempfehlung wird veröffentlicht, wenn Studiendaten zum derzeit durch die Europäische Arzneimittelbehörde mit bedingter Zulassung ausgestatteten Wirkstoff Bulevirtid verfügbar sind. Die Therapie der Hepatitis-C-Virus(HCV)-Koinfektion erfolgt analog zur HCV-Monotherapie, und es wird auf das aktuelle Addendum der Hepatitis-C-Leitlinie der DGVS verwiesen [10]. Wie auch unter interferonbasierter Therapie sollten bei HBsAg-positiven Patienten unter direkt wirksamer antiviraler Therapie (DAA) der HCV-Infektion zur Früherkennung einer HBV-Therapieindikation regelmäßige Kontrollen von HBV-DNA und Transaminasen erfolgen. Eine prophylaktische NA-Behandlung ist jedoch nicht erforderlich.

Hepatitis-B-Virusinfektion im Kindes- und Jugendalter: In diesem Kapitel erfolgte eine ausführliche Aktualisierung des Abschnitts zur antiviralen Therapie. So ist in Europa eine Therapie mit Tenofovir ab 12 Jahren, eine Therapie mit Entecavir ab 2 Jahren und eine Behandlung mit pegyliertem Interferon ab 3 Jahren zugelassen. Unverändert besteht eine Therapieindikation bei chronischer Hepatitis B, Therapieindikation und Behandlung 
sollten durch einen in diesem Gebiet erfahrenen Kinder-Gastroenterologen oder -Infektiologen erfolgen.

Zusammenfassend ist mit der aktuellen Leitlinie ein ausführliches Dokument zur Diagnostik, Behandlung und Therapie der Hepatitis-B-Virusinfektion erstellt worden. Evidenzbasierte Empfehlungen liefern Informationen für die an der Diagnostik und Therapie der HBV-Infektion beteiligten Berufsgruppen. Die Gültigkeitsdauer der Leitlinie wird zunächst auf 5 Jahre geschätzt. Im Fall von relevanten Neuerungen ist die Erstellung von Addenda geplant. So ist dies bereits für die Empfehlungen zur antiviralen Behandlung der HBV/HDV-Koinfektion vorgesehen. Ein entsprechendes Addendum wird nach Veröffentlichung der relevanten Studien erstellt werden.

\section{Interessenkonflikt}

Die Interessenkonflikte sind im Leitlinienreport angegeben.

\section{Literatur}

[1] Cornberg M, Protzer U, Dollinger MM et al. Prophylaxis, diagnosis and therapy of hepatitis B virus (HBV) infection: the German guidelines for the management of HBV infection. Z Gastroenterol 2007; 45 (12): 12811328

[2] Cornberg M, Protzer U, Petersen J et al. [Prophylaxis, diagnosis and therapy of hepatitis B virus infection - the German guideline]. Z Gastroenterol 2011; 49 (7): 871-930
[3] EASL 2017. Clinical Practice Guidelines on the management of hepatitis B virus infection. J Hepatol 2017; 67 (2): 370-398

[4] Terrault NA, Bzowej NH, Chang K-M et al. AASLD guidelines for treatment of chronic hepatitis B. Hepatology 2016; 63 (1): 261-283

[5] Terrault NA, Lok ASF, McMahon BJ et al. Update on prevention, diagnosis, and treatment of chronic hepatitis B: AASLD 2018 hepatitis B guidance. Hepatology 2018; 67 (4): 1560-1599

[6] Geneva: World Health Organization. Prevention of mother-to-child transmission of hepatitis B virus: guidelines on antiviral prophylaxis in pregnancy. 2020. Licence: CC BY-NC-SA 3.0 IGO. Available from: https:// apps.who.int/iris/bitstream/handle/10665/333391/9789240002708eng.pdf? sequence $=1$ \&isAllowed $=y$

[7] Greten TF, Malek NP, Schmidt S et al. Diagnostik und Therapie des hepatozellulären Karzinoms TT - Diagnosis of and Therapy for Hepatocellular Carcinoma. Z Gastroenterol 2013; 51 (11): 1269-1326

[8] EASL-ALEH. Clinical Practice Guidelines: Non-invasive tests for evaluation of liver disease severity and prognosis. J Hepatol 2015; 63 (1): 237-264

[9] Sarrazin C, Zimmermann T, Leberstiftung D. Deutsche Gesellschaft für Gastroenterologie VS (DGVS), (DGP) DG für P e. V et al. S3-Leitlinie „Prophylaxe, Diagnostik und Therapie der Hepatitis-C-Virus (HCV)Infektion“ TT - Prophylaxis, diagnosis and therapy of hepatitis-C-virus (HCV) infection: the German guidelines on the management of HCV infection. Z Gastroenterol 2018; 56 (7): 756-838

[10] Sarrazin C, Zimmermann T, Berg T et al. Prophylaxe, Diagnostik und Therapie der Hepatitis-C-Virus(HCV)-Infektion. Z Gastroenterol 2020; 58 (11): 1110-1131 